\title{
The Effect of Exercise on a Bicycle Ergometer with a Toe-Heel Pedal
}

\author{
Hiroshi NOBORI ${ }^{1)}$, Hitoshi MARUYAMA ${ }^{2)}$ \\ 1) Deptartment of Physical Therapy, International University of Health and Welfare, School of Nursing \\ and Rehabilitation Sciences at Odawara: 1-2-25 Shiroyama, Odawara-shi, Kanagawa-ken, 250-8588 \\ Japan. TEL: +81 465-21-6500, FAX: +81 465-21-6501 \\ 2) Deptartment of Physical Therapy, International University of Health and Welfare, School of Health \\ Sciences

\begin{abstract}
Purpose] The purpose of this study was to myoelectrically verify the effect of exercise on a bicycle ergometer with a newly developed toe-heel pedal. [Subjects and Methods] The subjects of this study were 12 healthy adults (24 limbs) without any disability in the lower limbs. We evaluated the integrated electromyograms (iEMG) of the quadriceps femoris and the flexor digitorum brevis during the pressing down on the toe-heel pedal and compared them with those recorded on the flat pedal. [Results] The average iEMG of the quadriceps femoris during the pedaling action was significantly higher for the toe-heel pedal at $5.49 \pm 3.06$ than that for the flat pedal at $1.75 \pm 2.17$. The average $i E M G$ of the flexor digitorum brevis during the pedaling action was higher for the toe-heel pedal at $2.39 \pm 0.73$ than that for the flat pedal at $1.86 \pm 1.83$. [Conclusion] We showed that exercise on an ergometer with the toe-heel pedal can effectively achieve facilitation of muscle contraction throughout the lower limb including the thighs as well as muscular training of the plantar muscles.

Key words: Toe-heel pedal, Ergometer, DYJOC
\end{abstract}

(This article was submitted Apr. 22, 2011, and was accepted Jun. 1, 2011)

\section{INTRODUCTION}

The authors developed a "toe-heel pedal exercise machine" for dynamic joint control training (DYJOC) for neuromotor coordination. The effect of exercise with the toe-heel pedal exercise machine was verified by examining the muscular activities of the quadriceps femoris (vastus medialis) (VM), which is regarded functionally as the most important muscle in the extension of the knee joint, and of the foot muscle (flexor digitorum brevis) ${ }^{1)}$ (FDB), which is regarded as regulating dynamic balance and posture control $^{2}$. In addition, we evaluated the effects of exercise based on indicators of proprioceptive sensation at the knee joint (threshold and joint position sense) ${ }^{3)}$. In the present study, we verified the effect of exercise on an ergometer with a new type of toe-heel pedal that we have recentry developed.

\section{SUBJECTS AND METHODS}

The subjects of this study were 12 healthy adults (24 limbs) without any disabilities in the lower limb (10 males and 2 females average age $21.2 \pm 2.7$ years, average height $167.9 \pm 9.8 \mathrm{~cm}$, and average weight $60.3 \pm 10.9 \mathrm{~kg}$ ). Subjects received adequate explanation about the study and provided their informed consent. Due consideration was given to ensuring that subjects would not suffer any disadvantages.
The toe-heel pedal was placed and fixed as the pedal part of the exercise ergometer (Fig. 1) so that the pressure was applied on subjects' toes and heels during pedaling. Since the load is applied to the toes and heel and the lengthwise arch of the inside and outside of the foot is kept up in the air, tension is created in FDB. As a result, the toeheel pedal, by its design structure, unavoidably induces isometric contraction in FDB during pedaling. The length between the loading stages for the toes and the heel is adjustable to the size of the foot. In order to facilitate measurement of myoelectric potential, a hole was made at the center of the pedal, through which wires connecting electrodes for the measurement of EMG were passed. A groove was also cut from the hole to one side of the pedal to accommodate the wires.

We measured the integrated electromyograms (iEMG) of VM and FDB during pressing down on the flat pedal (Fig. 1) and on the toe-heel pedal ergometer, and compared the average iEMG between the flat pedal and the toe-heel pedal. In order to evaluate the tension in VM during pedaling, we measured iEMG as the stable myoelectric activity per unit time instead of percentage of maximal voluntary contraction (\%MVC). In the statistical analysis, the paired t-test was used for the comparison of the iEMGs.

For both pedal types, the flat pedal and the toe-heel pedal, electromyograms were measured while isometric contraction was maintained for 10 seconds. The measurement item was iEMG which is calculated based on 


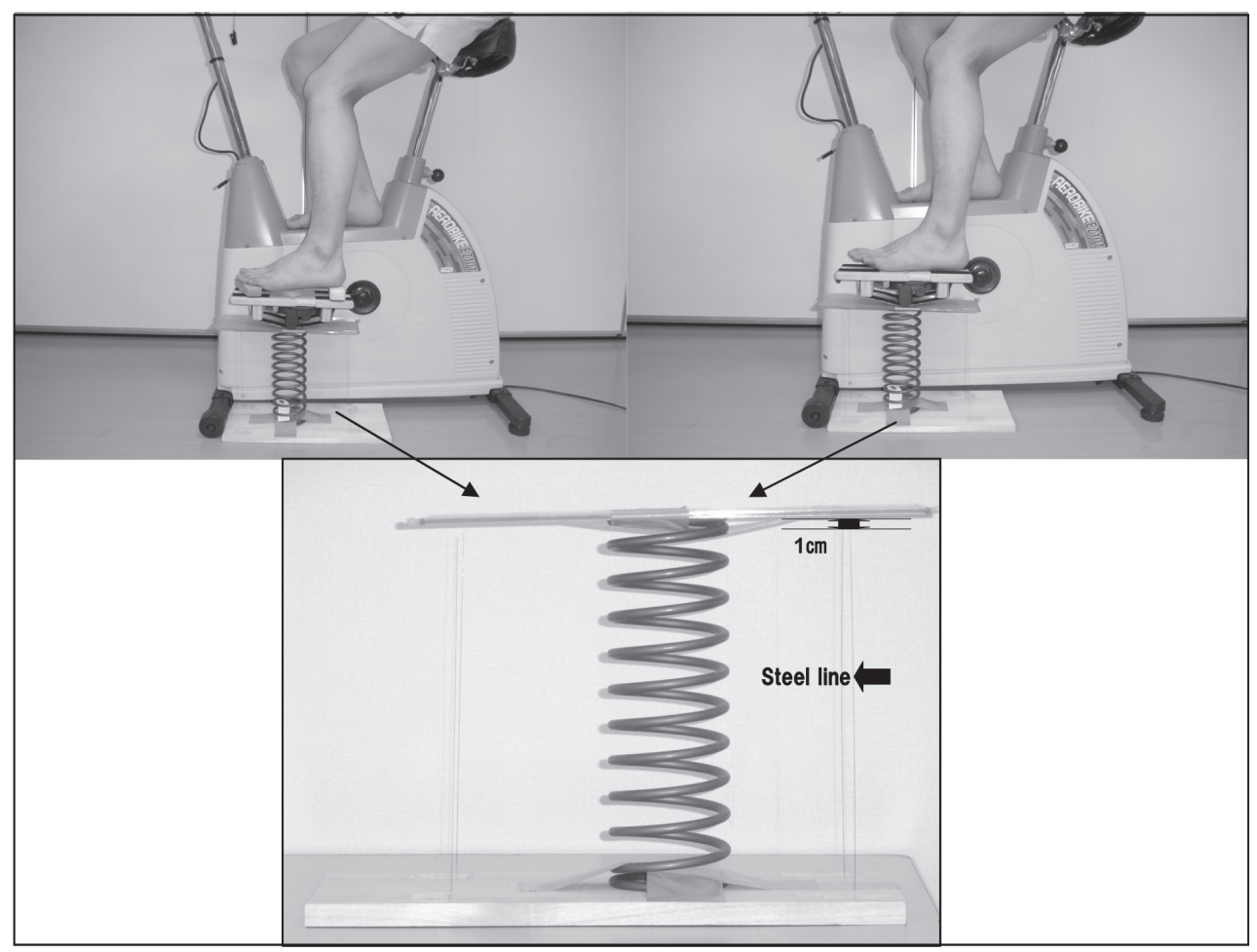

Fig .1 Pushing down on the toe-heel pedal and the flat pedal, and the load measurement table with a spring

the waveform of the electromyogram. The target muscles were VM and FDB. For the measurement, a laptop EMG measurement system PTS-137 (DKH Co., Ltd) and a portable data acquisition system BioLog DL-3100 system (DKH Co., Ltd) were used. The EMG signal was sampled at $500 \mathrm{~Hz}$ and iEMGs of stable contraction in an intermediate 2-second period were calculated and compared. Disposable electrodes (Blue sensor: Mets inc.) were placed according to the Kamori method ${ }^{4)}$, that is, four fingerwidths proximal from the superior medial border of the patella for the VM and the center of the line between the third metatarsal head and the calcaneus for FDB. Electrodes were applied on the middle of the muscles after adequate pretreatment at intervals of $2 \mathrm{~cm}$.

In order to ensure the power of pressing down on the pedal shaft from the anterior-horizontal position was $4.0 \mathrm{~kg}$, a load measurement table with a spring (Fig. 1) was constructed. The load measurement table was constructed by fixing a compression spring with a spring constant of 0.4 (Futaba Spring Industry) on a wooden baseboard (380 mm $\times 180 \mathrm{~mm} \times 15 \mathrm{~mm}$ ) and further fixing an acrylic board $(320 \mathrm{~mm} \times 120 \mathrm{~mm})$ on top of the spring. In order to determine the $10 \mathrm{~mm}$ horizontal descent of the acrylic board, four stainless wires (diameter $0.3 \mathrm{~mm}$; Taiho Trading Co., Ltd.) were attached to each of the four corners of the baseboard ensuring a distance of $10 \mathrm{~mm}$ between the top of the each wire and each of the four corners of the acrylic board. The pressing power of $4.0 \mathrm{~kg}$ was confirmed by visual confirmation of contact between the four stainless wires and the acrylic board by an observer.

The surface electromyograms were measured during pressing down on the flat pedal and on the toe-heel pedal with the subjects in the saddle seat position (knee joint extension $-45^{\circ}$ ). Subjects were instructed to maintain the power to press down the pedal shaft of the ergometer by 10 $\mathrm{mm}$ from the anterior-horizontal position $(4.0 \mathrm{~kg})$. The subjects were verbally cued at the start and the end of the 10 seconds of pressing down. The electromyogram was recorded for 10 seconds, and a stable record of 2 seconds some seconds after the start of the recording was selected for the analysis.

\section{RESULTS}

The averages of iEMG values of the 24 legs of the 12 subjects were compared. The average iEMG of VM, $5.49 \pm$ 3.06 , was significantly higher $(\mathrm{p}<0.001)$ for the toe-heel pedal than that for the flat pedal, $1.75 \pm 2.17$; and the average iEMG of FDB, $2.39 \pm 0.73$, was higher for the toeheel pedal than that for the flat pedal, $1.86 \pm 1.83$ (Table 1).

\section{DISCUSSION}

The purpose of the development of the toe-heel pedal for ergometers is to improve the efficiency of pedaling exercise on an ordinary ergometer and ultimately achieve the effect of DYJOC as a neuromotor coordination training. By DYJOC training, not only contraction of VM but also 
Table 1. Comparison of iEMGs between pushing down on the flat pedal and the toeheel pedal

\begin{tabular}{lcccr}
\hline & \multicolumn{2}{c}{ Vastus medialis (VM) } & \multicolumn{2}{c}{ Flexor digitorum brevis (FDB) } \\
\cline { 2 - 5 } & Flat pedal & T-H pedal & Flat pedal & T-H pedal \\
iEMG & $1.75 \pm 2.17$ & $5.49 \pm 3.06^{*}$ & $1.86 \pm 1.83$ & $2.39 \pm 0.73$ \\
\hline
\end{tabular}

$* \mathrm{p}<0.01$. average \pm standard deviation.

induction of simultaneous contraction of biceps femoris, anterior tibial muscle, and triceps surae through a closed kinetic chain $(\mathrm{CKC})$ can be expected ${ }^{5)}$. The toe-heel pedal was designed to induce isometric contraction in FDB through the tension created by applying load to the toes and heel with the lengthwise arch of the inside and outside of the foot kept in the air. In this condition, muscle strengthening exercise is unavoidable while pedaling.

The sole of humans forms an arch and tension is created in it by loading ${ }^{6}$. Loading of the five toes and the heel creates greater tension which strengthens the toe grip. Also, toe motion aiming to develop toe grip strength improves coordination through the activation of mechanoreceptors. Moreover, it induces chain motion of plantar muscles, gastrocnemius and hamstrings. On study ${ }^{7)}$ pointed out that toe motion improves and enhances postural control capacity. The purpose of DYJOC is to improve proprioceptive sensation. Another study ${ }^{8)}$ reported that the purpose of DYJOC is to enhance the stability of the lower legs against unexpected changes in the direction or speed of lower leg motion.

Expected exercise effects arising from use of an ergometer with the toe-heel pedal are as follows.

I. Muscle strengthening exercise by isometric contraction of plantar muscles $\downarrow$

II. Muscle strengthening exercise of the VM $\downarrow$

III. DYJOC effects $\downarrow$

IV. Coordination exercise effects of the legs as a whole

In the present study, we compared the average iEMGs of 24 legs of 12 subjects in pressing down on the toe-heel pedal and the flat pedal attached to an ergometer. The target muscles were VM and FDB. The average iEMGs of VM and FDB between pressing down on the toe-heel pedal and on the flat pedal were higher for the toe-heel pedal for both of the muscles and the result was especially significant $(p<0.01)$ for VM. The design of the toe-heel pedal induces tension in FDB and greater muscle activity is expected. Also, CKC effect is expected, which we consider greatly increased the inductive effect on the lower limbs as a whole.

Based on these results, we consider that the pedaling action on the toe-heel pedal induces greater muscle activity in FDB and greatly induces contraction of VM, while the pedaling action on the flat pedal induced smaller muscle activity of in FDB and tended to induce smaller muscle activity in VM.

It has been reported that there is a linear relationship between $\mathrm{iEMG}$ and tension ${ }^{9,10)}$. Another study reported that there is a linear relationship between iEMG and maximum voluntary contraction up to $80 \%$ of maximum voluntary contraction $^{11)}$. Considering these reports, we expect that a higher iEMG value indicates more intense muscle strengthening exercise. In other words, pedaling action with the toe-heel pedal allows muscle strength training of not only the plantar part but also the whole lower limb including the thigh.

We conclude that pedaling action on an ergometer fitted with the toe-heel pedal is useful as a DYJOC exercise and serves as a coordination exercise for each joint in the lower limb.

The measurements in this study were conducted using a 2-channel EMG. In the future, the effect on not only the $\mathrm{VM}$ and the FDB, but also on other muscles should be examined.

\section{REFERENCES}

1) Kito $\mathrm{N}$, Ihara $\mathrm{H}$, Miwa $\mathrm{K}$, et al.: Effect of the toe training as the elderly person fall prevention. Physical Therapy Japan, 2001, 7: 313-319.

2) Nobori H, Maruyama H, Takahashi N: Development of the Toe-Heel pedal device-Study of the iEMG-. J Phys Ther Sci, 2004, 19: 351-356.

3) Nobori H, Maruyama H, Takahashi N: The effect of a toe- heel pedal exercise machine. J Phys Ther Sci, 2006, 18: 143-148.

4) Kamori R: Anatomy guide for iEMG (3rd ed). Nigata: Nishimura Shyoten. 2003, pp120-121, pp190-191.

5) Kawamura K: Open kinetic chain and closed kinetic chain. J Clin Reha, 1996, 15: 186-189.

6) Nagata A: Biomechanics of Bones and Joints. J Phys Ther, 1995, 29: 514 518.

7) Ihara H: Joint Training (The revision 2nd ed). Neuro-Coordinatinally exercise; Tokyo: KYODOUISYO Syuppansya. 2000, pp 191-197.

8) Bunton EE, Pitny WA, Kane AW, et al.: The role of limb torque muscle action and proprioception during closed kinetic chain rehabilitation of the lower extremity. J Athletic Train, 1993, 28: 23-30.

9) Lippold OC: The relation between integrated action potentials in human muscle and its isometric tension. J Physiol, 1952, 177: 492-499.

10) deVries HA: Relationship of resting EMG level to total body metabolism with reference to the origin of "tissue noise". Am J Phys Med, 1976, 55: 139-147.

11) Kuroda E, Klissouras V, Milsum JH: Electricl and metabolic activities and Fatigue in human isometric contraction. J Appl Physiol, 1970, 29: 358-367. 